\title{
Refinement Method for Weighting Scheme of Fully Spatial Beamformer
}

\section{Chayanit Bunsanit, Peerapong Uthansakul, and Monthippa Uthansakul}

School of Telecommunication Engineering, Suranaree University of Technology, Muang, Nakhon Ratchasima 30000, Thailand

Correspondence should be addressed to Monthippa Uthansakul, mtp@sut.ac.th

Received 10 April 2012; Revised 28 May 2012; Accepted 7 June 2012

Academic Editor: Chih-Peng Li

Copyright ( $\odot 2012$ Chayanit Bunsanit et al. This is an open access article distributed under the Creative Commons Attribution License, which permits unrestricted use, distribution, and reproduction in any medium, provided the original work is properly cited.

So far, a wideband spatial beamformer has been proposed. This kind of beamformer has a major contribution as its weighting coefficients are real valued in which they can be simply realized by attenuators or amplifiers. However, so far, the range of attenuation or amplification is relatively large which is not practical for hardware realization. Therefore, this paper proposes a concept to reduce the range of weighting coefficients hence, the hardware realization becomes practical. In this paper, a full prototype of wideband spatial beamformer is constructed to reflect the true beamforming performance of the proposed refinement method. Its radiation patterns obtained from simulation and measurement are compared. As a result, we can reduce the attenuation or amplification range while some radiation characteristic is remained.

\section{Introduction}

In recent years, wireless communication systems have grown rapidly and contained several technologies operating in different frequencies, for example, WiFi, WiMAX, Bluetooth, and broadband WLAN. From these reasons, the systems are demanded for better coverage, wider frequency range, higher quality of service, and more capacity [1]. So far, smart antenna systems have been envisaged to be the solution. This is because the systems can form one beam towards a desired direction and create nulls or sidelobes towards interference directions. As a result, the greatly improving system performance and also saving energy can be obtained [2]. The smart antenna systems usually consist of antenna array and a suitable signal-processing unit adjusting the weighting coefficients at individual antenna elements to ease the effect of interference signal [3]. The signal processing works according to the utilized algorithms. So far, the beamforming algorithms for smart antennas in wireless communications have concerned only narrowband operation. However, in order to support high data-rate transmission, new efforts are currently required to deal with smart antennas in wide frequency range [4]. From literatures [5-7], wideband smart antenna technology can be classified into three categories. The first category utilizes space-time signal processing, so called spatiotemporal beamformer. These systems consist of array antennas and tapped-delay line at each branch of the array to deal with the received signal in time domain [8]. However, the number of tapped-delay line increases with the bandwidth of utilized frequency. Next category relies on space-frequency signal processing. This is an alternative approach to perform wideband beam-formation without the use of tapped-delay lines. In this method the received signal will be decomposed into non-overlapping narrowband component using band-pass filter. However, for wideband signal, the systems require a large number of frequency filters $[9,10]$. The last category utilizes only spatial signal processing, so-called wideband spatial beamformer. This is a new method for wideband smart antennas. In the part of signal processing, weighting coefficients are calculated using two-dimensional Inverse Discrete Fourier Transform (IDFT) technique applied to the required radiation pattern. The obtained weighting coefficients become real values which can be simply realized by attenuators or amplifiers [11-13]. The main advantage of this wideband spatial beamformer is that its design does not require phase shifters, delay circuits, or 


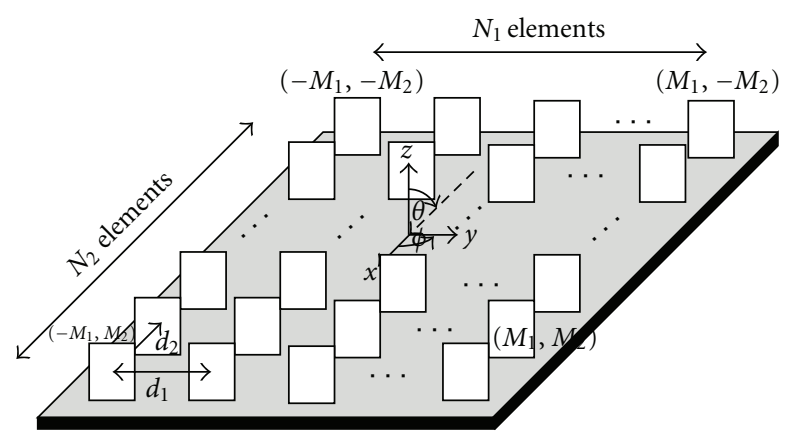

FIGURE 1: Layout of array configuration for fully spatial beamformer.

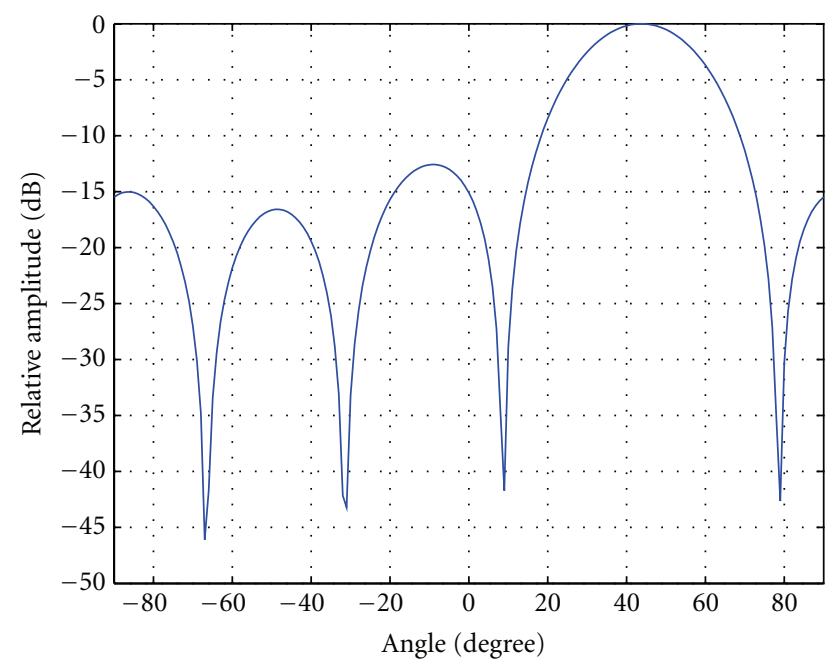

— IDFT method (sinc function)

FIgURE 2: Simulated radiation pattern of $4 \times 4$ beamformer at $2.15 \mathrm{GHz}$, when the main beam direction is $45^{\circ}$.

frequency filters. This is of considerable practical advantage over the other types of beamformer employing complex weighting coefficients [14]. However, the range between maximum and minimum values of weighting coefficients (attenuation or amplification) is relatively wide. This is considerably impractical. Therefore, this paper proposes the refinement method for fully spatial beamformer. The aim of the proposed method is to reduce the range of overall weighting coefficients. As a result, the implementation of this kind of beamformer is practical. A full prototype of fully spatial beamformer is developed in order to validate the proposed method.

The remainder of this paper is as follows. After brief introduction showing the background and motivation of the paper, the concept of beam formation in wide range of operating frequency band using only spatial signal processing is described in Section 2. Section 3 proposes the method to make the weights more practical, so-called refinement method. A full prototype of fully spatial beamformer is detailed and constructed in Section 4. Then, its beamforming

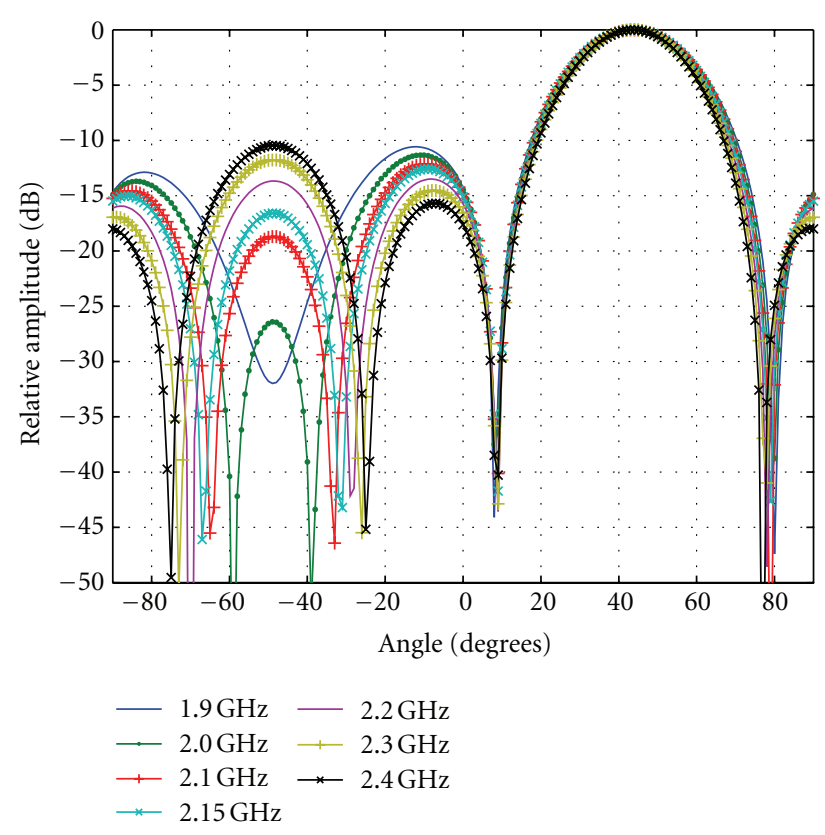

FIGURE 3: Simulated radiation pattern of $4 \times 4$ beamformer for frequencies from 1.9 to $2.4 \mathrm{GHz}$, when the main beam direction is $45^{\circ}$.

performance is revealed with the use of proposed refinement method in Section 5. Finally, Section 6 concludes the article.

\section{Wideband Beamforming Algorithm Using Fully Spatial Signal Processing}

2.1. Fully Spatial Beamformer. The configuration of a wideband beamformer employing a fully spatial signal processing for beam and null steering in azimuth direction is shown in Figure 1. It is constituted by $N_{1} \times N_{2}$ where $d_{1}$ and $d_{2}$ represent array spacing in two orthogonal directions and are usually chosen as half-wavelength $(\lambda / 2)$ at the highest frequency of a given frequency band. The antenna elements are denoted by indices $\left(m_{1}, m_{2}\right)$ where $-M_{1} \leq m_{1} \leq M_{1}$ and $-M_{2} \leq m_{2} \leq M_{2}$. The relationship between $M$ and $N$ is $M_{i}=\left(N_{i}-1\right) / 2[15]$.

The radiation pattern of the array as a function of angle and frequency can be given by

$$
\begin{array}{r}
H(f, \phi)=G(f, \phi) \\
\cdot \sum_{m_{1}=-M_{1}}^{M_{1}} \sum_{m_{2}=-M_{2}}^{M_{2}} w_{m_{1}, m_{2}} \\
\cdot e^{j(2 \pi f / c)\left(d_{1} m_{1} \sin \phi+d_{2} m_{2} \cos \phi\right)},
\end{array}
$$

where $f$ is the frequency variable, $c$ is the speed of signal, and $G(f, \phi)$ is a function of frequency-angle-dependent gain of each antenna element. Note that signal is incident or transmitted in azimuth direction $\phi$ and $\theta=90^{\circ}$. When $\phi$ and $\theta$ are the azimuth and the elevation angles, respectively. 
In order to determinate the weighting coefficients $w_{m_{1}, m_{2}}$ in (1), a modified IDFT is applied to $H$ as shown in (2)

$$
\begin{aligned}
& w_{m_{2}, m_{2}}=\left(\frac{1}{N_{u_{1}} \cdot N_{u_{2}}}\right) \\
& \times \sum_{u_{1}=-0.5}^{0.5} \sum_{u_{2}=-0.5}^{0.5} \frac{H\left(u_{1}, u_{2}\right)}{G\left(u_{1}, u_{2}\right)} \\
& \quad \times e^{-j 2 \pi u_{1} m_{1}} e^{-j 2 \pi u_{2} m_{2}} .
\end{aligned}
$$

We define two auxiliary functions as $u_{1}=\left(f d_{1} / c\right) \sin \phi$ and $u_{2}=\left(f d_{2} / c\right) \cos \phi$. Where $N_{u_{1}}$ and $N_{u_{2}}$ are number of sampling points in $u_{1}-u_{2}$ plane in which $N_{u_{1}} \cong 2 N_{1}$ and $N_{u_{2}} \cong 2 N_{2}$. The $H\left(u_{1}, u_{2}\right)$ is the form of Sinc function to obtain maximum gain in the desired direction [16] which can be given as

$$
H\left(u_{1}, u_{2}\right)= \begin{cases}\frac{\sin \left[\alpha \pi\left(u_{1} / u_{2}\right)-\tan \phi_{0}\right]}{\alpha \pi\left(u_{1} / u_{2}\right)-\tan \phi_{0}}, & r_{l}<|r|<r_{h}, \\ \frac{1}{\sqrt{10}}, & \text { otherwise }\end{cases}
$$

where $\phi_{0}$ is the direction at which the main beam to be pointed to which is assumed to be prior known [14], $\alpha$ is the number related to the main beam first-null width, $r_{l}$ and $r_{h}$ are defined by the following expressions: $r_{l}=\left(f_{l} / c\right) \bar{d}$ and $r_{h}=\left(f_{h} / c\right) \bar{d}$, respectively, when $\bar{d}=$ $\sqrt{d_{1}^{2} \sin ^{2} \phi_{0}+d_{2}^{2} \cos ^{2} \phi_{0}}, f_{l}$ is lowest frequency and $f_{h}$ is highest frequency. Referring to the previous work presented in [14], null steering implementation is difficult to perform using IDF method. This is because the obtained weighting coefficients become real valued. Therefore, degree of freedom is relatively limited comparing the ones employing complex weighting coefficients.

To give some simulation examples, we consider $4 \times 4$ array operating over frequencies from 1.9 to $2.4 \mathrm{GHz}$ and choose planar monopole having omnidirectional radiation to be an antenna element. From (1) to (3), we calculate weighting coefficients when the main beam is required to be pointed to $\phi_{0}=45^{\circ}$. The outcomes of beam formation are shown in Figures 2 and 3.

Figures 2 and 3 show the radiation pattern of the $4 \times 4$ spatial beamformer in which the objective function as shown in (3) is assumed using Sinc function with the mainbeam pointed to $45^{\circ}$ off the array broadside direction. These figures indicate that the main beams are correctly directed towards the desired direction, and the patterns are alike throughout the desired frequency band, from 1.9 to $2.4 \mathrm{GHz}$. Also, it can be observed that as frequency increases, the array's sidelobe levels increase, and the null locations slightly change with respect to the operating frequency. However, from the results, the validity of the wideband spatial beamforming concept can be confirmed.

2.2. Chebyshev Function. In the previously section, we consider the Sinc function being as an objective function $H\left(u_{1}, u_{2}\right)$. As a result, the sidelobe level (SLL) is relatively

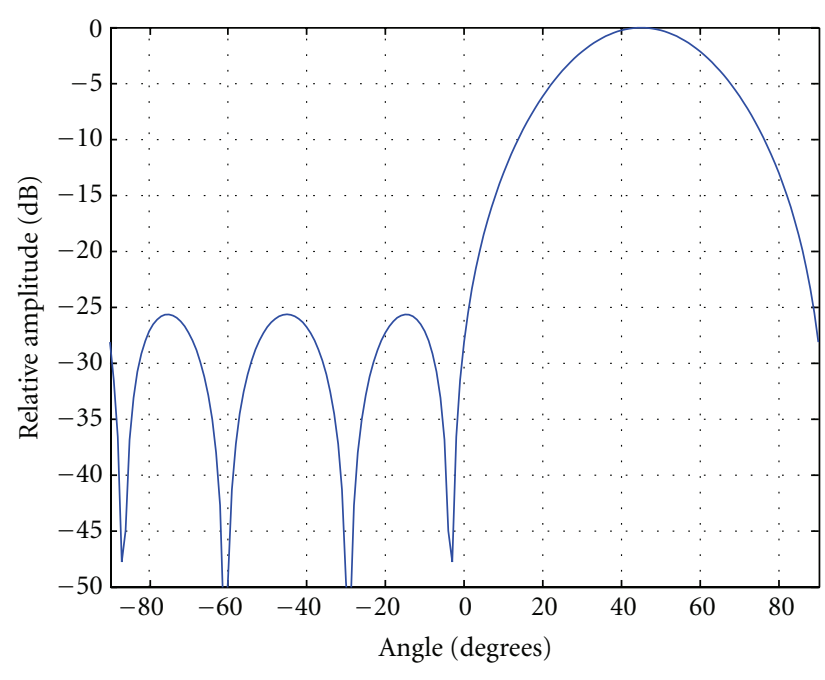

— IDFT method (chebyshev function)

FIGURE 4: Simulated radiation pattern of $4 \times 4$ beamformer plotted at $2.15 \mathrm{GHz}$, when the main beam direction is $45^{\circ}$, and the objective function is Chebyshev.

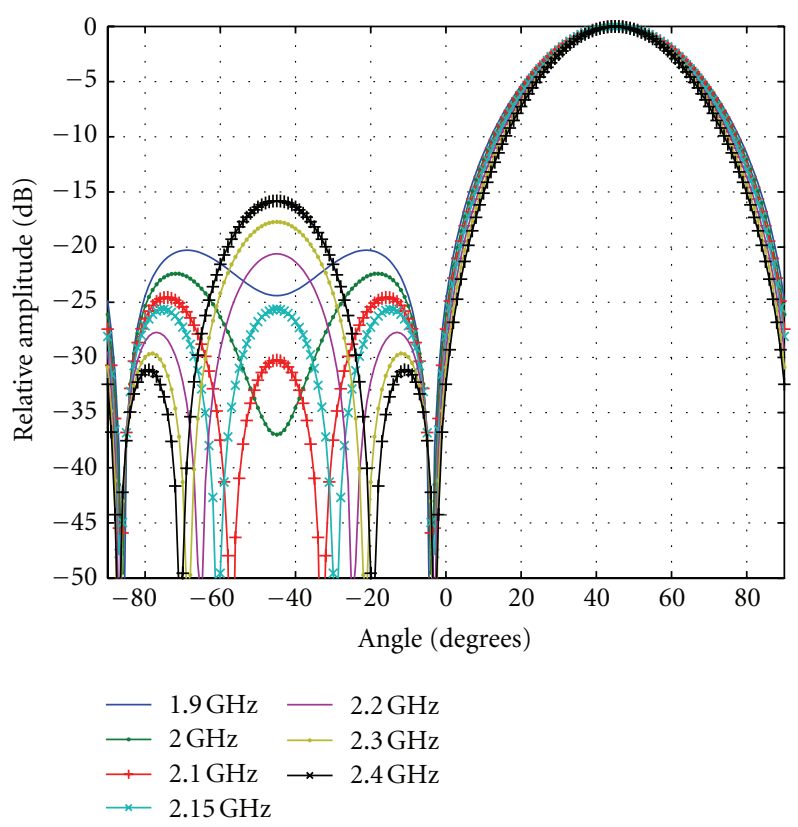

FIGURE 5: Simulated radiation pattern of $4 \times 4$ beamformer for frequencies from 1.9 to $2.4 \mathrm{GHz}$, when the main beam direction is $45^{\circ}$, and the objective function is Chebyshev.

high. Thus, in this section, the objective function is created, using Chebyshev function instead as shown in (4) [17, 18]. We consider

$$
H\left(u_{1}, u_{2}\right)= \begin{cases}X, & \text { desired frequency band } \\ \frac{1}{\sqrt{10}}, & \text { otherwise }\end{cases}
$$


where

$$
\begin{gathered}
X= \begin{cases}(-1)^{N} \cosh (N \cdot \operatorname{arccosh}|x|), & x<-1 \\
\cos (N \cdot \arccos x), & |x| \leq 1 \\
\cosh (N \cdot \operatorname{arccosh} x), & x \geq 1,\end{cases} \\
x=x_{0} \cos \left\{\frac{2 \pi}{\lambda} d \sin \frac{(\theta)}{2}\right\}, \\
x_{0}=\cosh \left(\frac{\cosh { }^{-1} \operatorname{SLL}_{\mathrm{dB}}}{N}-1\right), \\
N=N_{1}=N_{2} .
\end{gathered}
$$

From (6) direction of arrival on $u_{1}-u_{2}$ plane is

$$
\theta=a \tan \left(\frac{u_{1}}{u_{2}}-\phi_{0}\right)
$$

Substituting (9) into (6) yields

$$
x=x_{0} \cos \left(\frac{2 \pi}{\lambda} d \sin \frac{\left(a \tan \left(u_{1} / u_{2}\right)-\phi_{0}\right)}{2}\right)
$$

or

$$
x=x_{0} \cos \left(\pi \sin \frac{\left(a \tan \left(u_{1} / u_{2}\right)-\phi_{0}\right)}{2}\right) .
$$

In this section, it is easily done by replacing the objective function $H\left(u_{1}, u_{2}\right)$ appeared in (3) by the one appeared in (4). Therefore, SLL is expected to be lower comparing to the ones shown in Figures 2 and 3. The simulation results for this assumption are shown in Figures 4 and 5.

Comparing between Figures 4 and 5 and Figures 2 and 3 reveals that the sidelobe level when objective function is assumed using Chebyshev polynomial is lower than using Sinc function for all frequencies. In Figure 5, the null location slightly changes when frequency increases as the ones appeared in Figure 3.

\section{Refinement Method for Weighting Scheme of Fully Spatial Beamformer}

Regarding the previous section, it has shown that we can obtain a value of weighting coefficient of smart antenna by taking IDFT to the desired radiation pattern. One example of the obtained weighting coefficients using both Sinc and Chebyshev functions is shown in Table 1. As we can see they are all real valued in which we can simply utilize attenuators or amplifiers to be weights. However, as we can notice in Table 1, they are not integer numbers, and also the range between the maximum and minimum values is relatively wide. This is considerably not practical for hardware implementation. Therefore, the modification of calculated weighting coefficients is needed while maintaining some important characteristic of radiation pattern.

In this section, we describe the proposed refinement method for weighting scheme of fully spatial beamformer in order to make it practical. This method is implemented after initially calculating the weighting coefficients using IDFT as mentioned before. Some significant properties of radiation behavior are maintained as described below.
3.1. Refinement Algorithm. The main proposal of this algorithm is to decrease the operation range of weighting coefficients as an example shown in Table 1. This algorithm is required because simply squeezing the mentioned range affects some importance radiating characteristics such as main-beam's direction and sidelobe levels. However, obtaining the integer numbers in weighting coefficients is byproduct when performing refinement method.

The weighting coefficient from IDFT method detailed in Section 2.1 is now defined as

$W_{o}=\left\{w_{o, 1}, w_{o, 2}, w_{o, 3}, \ldots, w_{o, N}\right\}=\left\{w_{o, i}\right\}, \quad i=1,2,3, \ldots, N$,

where $N$ is number of array antennas. Usually, the value of $W_{o}$ is general number, then we round the weights obtained from IDFT method to integer-valued weights $\left(W_{r}\right)$ as

$$
W_{r}=\left\{w_{r, 1}, w_{r, 2}, w_{r, 1}, \ldots, w_{r, N}\right\}=\left\{w_{r, i}\right\}=\operatorname{Int}\left\{w_{o, i}\right\} .
$$

Then, the required mainbeam direction $\left(\phi_{0}\right)$, average level of minor lobe $\left(\mathrm{MLL}_{0}\right)$ and width of main beam $\left(\delta_{0}\right)$ and weighting coefficient $\left(W_{r}\right)$ are given. Next, we carry out the following steps.

(1) Determine the maximum of $W_{r}$, that is

$$
w_{\max }^{(k)}=\underset{i=1}{\max }\left\{w_{r, i}\right\},
$$

where $k=1,2,3 \ldots$ is order of iteration.

(2) Decrease maximum of weighting coefficient $\left(w_{\max }^{(k)}\right)$ by decreasing a step size, where $\Delta w>0$. This results in integer number for weighting coefficients. We have

$$
w_{\max , j}^{(k)}=\left\|w_{\max }^{(k)}-\Delta w\right\|
$$

where $j=1,2,3, \ldots$ Then we replace $w_{\max , j}^{(k)}$ with $w_{\max }^{(k)}$ in a set of $W_{r}$. We consider

$$
w_{\max , j}^{(k)} \Longrightarrow w_{\max }^{(k)}
$$

(3) Multiply $W_{r}$ by steering vector $\psi$, and we can get output as follows:

$$
\begin{gathered}
y_{j}^{(k)}=\sum_{i=1}^{N} w_{r, i} e^{\psi}, \\
y_{j}^{(k)}=\sum_{i=1}^{N} w_{r, i}\left(\exp \left(\left(\frac{2 \pi}{c}\right)\left(d_{1} p \sin \phi+d_{2} q \cos \phi\right)\right)\right),
\end{gathered}
$$

where $(p, q)$ is index of antenna elements.

(4) Now, we can obtain the radiation pattern from (18). Then we can check some significant parameters such as mainbeam direction $\left(\phi_{r}^{(k)}\right)$, average minor-lobe level 
TABLE 1: Weighting coefficients when the required radiation pattern is created using Sinc and Chebyshev functions.

\begin{tabular}{|c|c|c|c|c|c|}
\hline \multirow{3}{*}{ Number of element } & \multicolumn{2}{|c|}{ Attenuation (dB) } & \multirow{3}{*}{ Number of element } & \multicolumn{2}{|c|}{ Attenuation (dB) } \\
\hline & \multicolumn{2}{|c|}{ Objective } & & & ve \\
\hline & Sinc & Chebyshev & & Sinc & Chebyshev \\
\hline 1 & 11.72 & 22.78 & 9 & 22.82 & 21.47 \\
\hline 2 & 9.68 & 6.83 & 10 & 0 & 0 \\
\hline 3 & 11.72 & 21.47 & 11 & 11.98 & 16.80 \\
\hline 4 & 0.94 & 5.87 & 12 & 5.50 & 6.83 \\
\hline 5 & 5.50 & 6.83 & 13 & 0.94 & 5.87 \\
\hline 6 & 11.98 & 16.80 & 14 & 11.72 & 21.47 \\
\hline 7 & 0 & 0 & 15 & 9.68 & 6.83 \\
\hline 8 & 22.82 & 21.47 & 16 & 11.72 & 22.78 \\
\hline
\end{tabular}

TABle 2: Parameters given in simulation for refinement algorithm of 6 cases.

\begin{tabular}{lcccccc}
\hline \multirow{2}{*}{ Parameters } & \multicolumn{5}{c}{ Cases of refinement } \\
& I & II & III & IV & V & VI \\
\hline$\Delta \phi$ (degrees) & 0 & 1 & 2 & 3 & 4 & 4 \\
$\Delta$ MILL (dB) & 0 & 1 & 2 & 3 & 4 & 4 \\
$\Delta \delta$ (degrees) & 0 & 1 & 2 & 3 & 4 & 5 \\
Number of directions & 67 & 71 & 77 & 86 & 90 & 91 \\
\hline
\end{tabular}

$\left(\mathrm{MLL}_{r}^{(k)}\right)$, and beamwidth $\left(\delta_{r}^{(k)}\right)$ according to the following conditions:

$$
\begin{gathered}
w_{\max }^{k} \leq w_{0}, \\
\phi-\Delta \phi \leq \phi_{r}^{(k)} \leq \phi+\Delta \phi, \\
\mathrm{MLL}_{r}^{(k)} \leq \mathrm{MLL}_{0}+\Delta \mathrm{MLL}, \\
\delta_{0}-\Delta \delta \leq \delta_{r}^{(k)} \leq \delta_{0}+\Delta \delta,
\end{gathered}
$$

where $w_{0}$ is desired maximum of weighing coefficients, $\Delta \phi$ is deviation of main beam angle, $\Delta$ MLL is deviation of average minor-lobe level, $\Delta \delta$ is deviation of beamwidth.

(5) Repeat steps 2 to 4 until the set of weighting coefficient is zero according to (19) to (22), and then we can obtain a set of new solution $\left(W_{r}\right)$ for the refinement method.

This proposed algorithm can run in both offline or real time processing depending on the purpose of users. In case of ruing in real-time, we need a suitable DSP board to receive the important information from DFT method then perform 5 steps refinement as shown above. Afterwards, the DSP board feedbacks the weighting coefficients to control the attenuators or amplifiers.

3.2. Simulation Results. In this section we show the efficiency of the proposed refinement method using our own developed program in MATLAB. The utilized antennas are arranged in $4 \times 4$ lattice, and the operating frequency is given from 1.9 to $2.4 \mathrm{GHz}$. We also assume the decreasing step size of weighting coefficient of 1 , desired maximum of weight value of $32 \mathrm{~dB}$, and steering angle from 0 to 90 degrees. Some parameters are given in simulations for 6 cases as shown in Table 2.

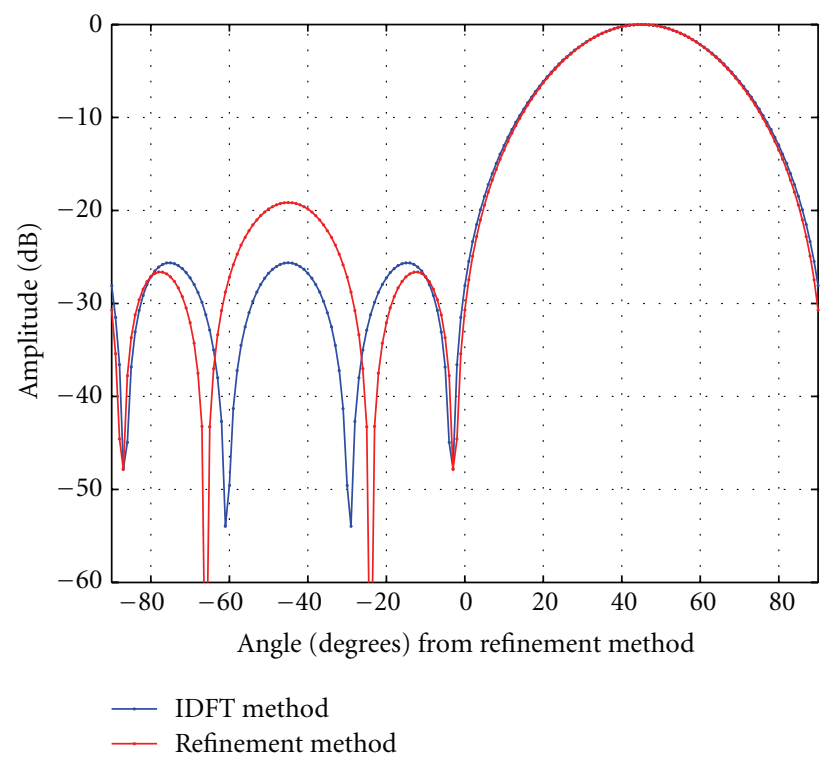

FIGURE 6: The comparison of radiation patterns at $45^{\circ}$ using IDFT method and Refinement method at frequency $2.15 \mathrm{GHz}$.

Table 2 shows 6 cases for the proposed refinement method. Some parameters, for example, maximum weighing coefficients, deviation of mainbeam angle $(\Delta \phi)$, deviation of average minor lobe level ( $\triangle \mathrm{MLL}$ ), and deviation of beamwidth $(\Delta \delta)$ are differently given in each case. For case I, we set the conditions not to have any error in the parameters mentioned earlier. From running some simulations, we have found that 67 directions or cases out of 91 directions, $0^{\circ}-$ $90^{\circ}$, are succeeded for refinement. This means that there are only 67 directions out of 91 directions in which we can maintain no error in mainbeam direction, minor lobe level and beamwidth. For case II, we allow the slight error in mainbeam direction, minor lobe level and beamwidth as shown in Table 2. We have found that the number of success cases increases from 67 to 71 directions. Also, higher error in mainbeam direction, minor lobe level, and beamwidth is allowed as shown in Table 2 for cases III, IV, V, and VI. As we can see, the number of success cases increase as we allow more error in mainbeam direction, minor-lobe level 
TABLE 3: The comparison value of attenuator factors between IDFT method and refinement method.

\begin{tabular}{lccccc}
\hline & \multicolumn{2}{c}{ Attenuation $(\mathrm{dB})$} & \multicolumn{2}{c}{ Attenuation $(\mathrm{dB})$} \\
Number of element & IDFT & Refinement & Number of element & IDFT & Refinement \\
\hline 1 & 22.78 & 18 & 9 & 21.47 & 18 \\
2 & 6.83 & 7 & 10 & 0 & 0 \\
3 & 21.47 & 18 & 11 & 16.80 & 17 \\
4 & 5.87 & 6 & 12 & 6.83 & 7 \\
5 & 6.83 & 7 & 13 & 5.87 & 6 \\
6 & 16.80 & 17 & 14 & 21.47 & 18 \\
7 & 0 & 0 & 15 & 6.83 & 7 \\
8 & 21.47 & 18 & 16 & 22.78 & 18 \\
\hline
\end{tabular}

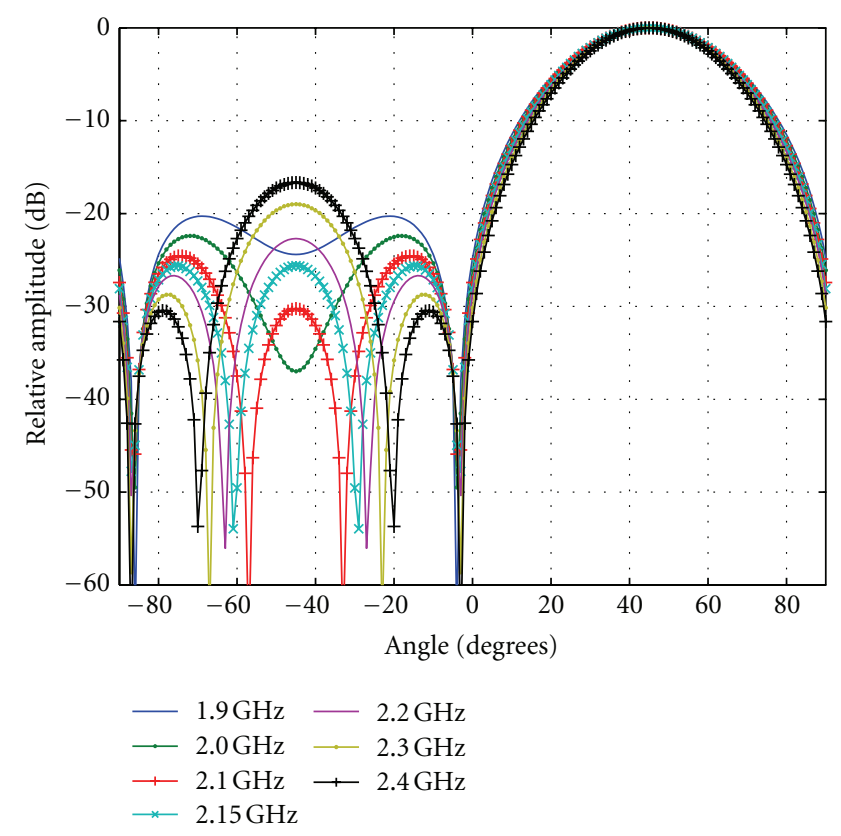

FIGURE 7: Radiation pattern at $45^{\circ}$ using IDFT method from frequencies 1.9 to $2.4 \mathrm{GHz}$.

and beamwidth. For case VI, 100\% success can be achieved when we allow error in mainbeam direction of $4^{\circ}$, minor lobe level of $4^{\circ}$ and beamwidth of $5^{\circ}$. For this case, the maximum weighting coefficients can be decreased from $54 \mathrm{~dB}$ to $32 \mathrm{~dB}$.

Table 3 shows 16 values of weighting coefficients comparing between IDFT method and proposed refinement method when the desired mainbeam direction is given at $45^{\circ}$. For the refinement method, we set $w_{0}=18 \mathrm{~dB}, \Delta \phi=0^{\circ}$, $\Delta \mathrm{MLL}=3 \mathrm{~dB}$ and $\Delta \delta=0^{\circ}$. As we can see in Table 3, we can decrease maximum weighting coefficient from $22.78 \mathrm{~dB}$ to $18 \mathrm{~dB}$ which mean the range of attenuation or amplification decreases.

Figure 6 shows beamforming performance for case shown in Table 3 at frequency $2.15 \mathrm{GHz}$ comparing between using IDFT method and proposed refinement method. The obtained result shows that after the refinement method is done, the mainbeam direction is still pointed to $45^{\circ}$, and there is no error in beamwidth. Also, the error in average minor lobe level is within $3 \mathrm{~dB}$.

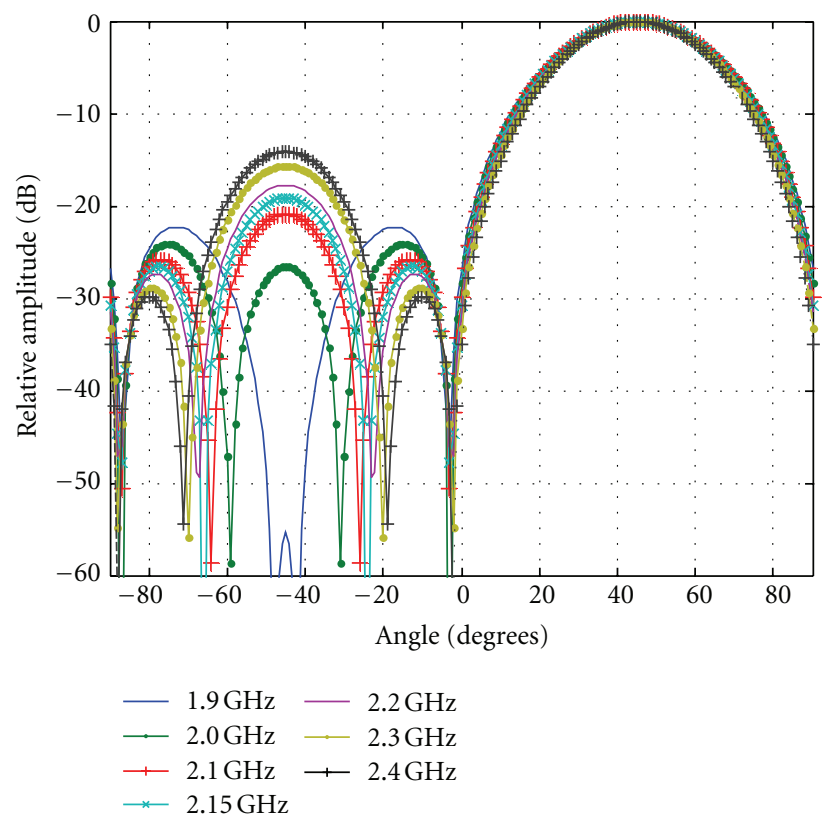

FIGURE 8: Radiation pattern at $45^{\circ}$ using proposed refinement method from frequencies 1.9 to $2.4 \mathrm{GHz}$.

Figures 7 and 8 show beamforming performance of the beamformer throughout the designated band using IDFT method and proposed refinement method, respectively. As we can see, the beamforming performance for both cases is similar while we can decrease the range of attenuation or amplification up to $4.78 \mathrm{~dB}(22.78-18 \mathrm{~dB})$.

\section{Practical Realizations}

The full prototype employing $4 \times 4$ array antennas for the proposed antenna systems is designed for the operating frequencies from 1.9 to $2.4 \mathrm{GHz}$ as shown in Figure 9. The prototype is a digitally controlled analog beamforming system, and it consists of three functional blocks: antenna array, weighting network, and control devices. A block diagram of smart antenna systems starts from the received incident signals at $4 \times 4$-antenna array. The received signals are weighted in weighting network which is controlled 


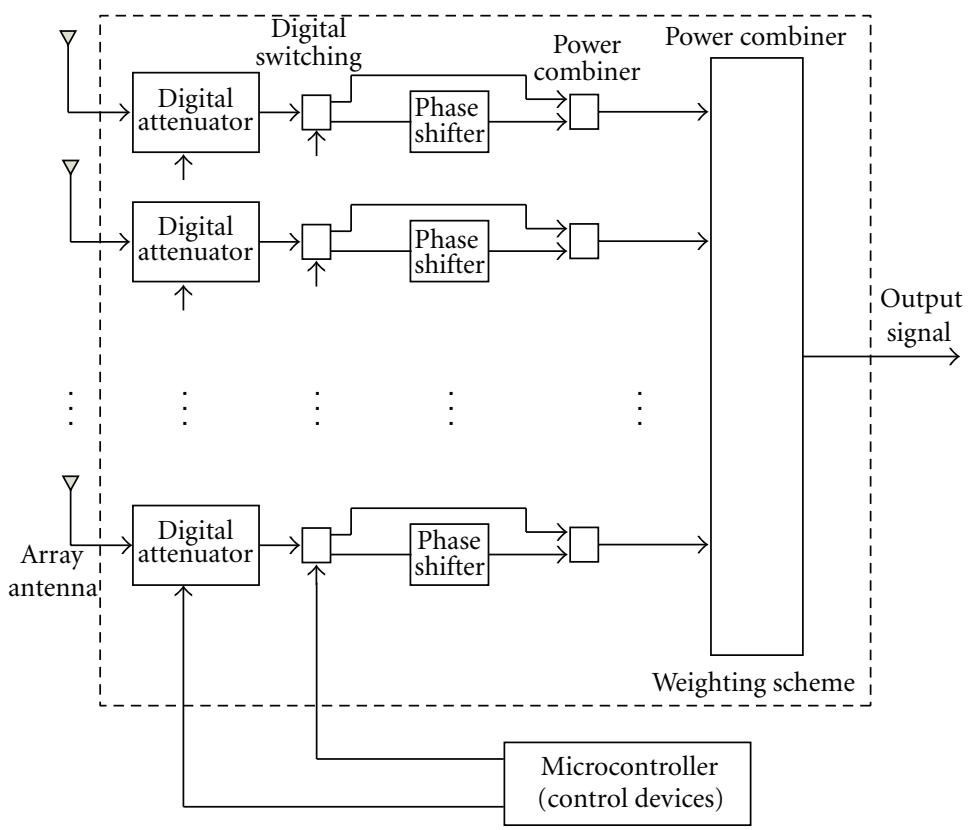

FIGURE 9: Diagram of smart antenna system.

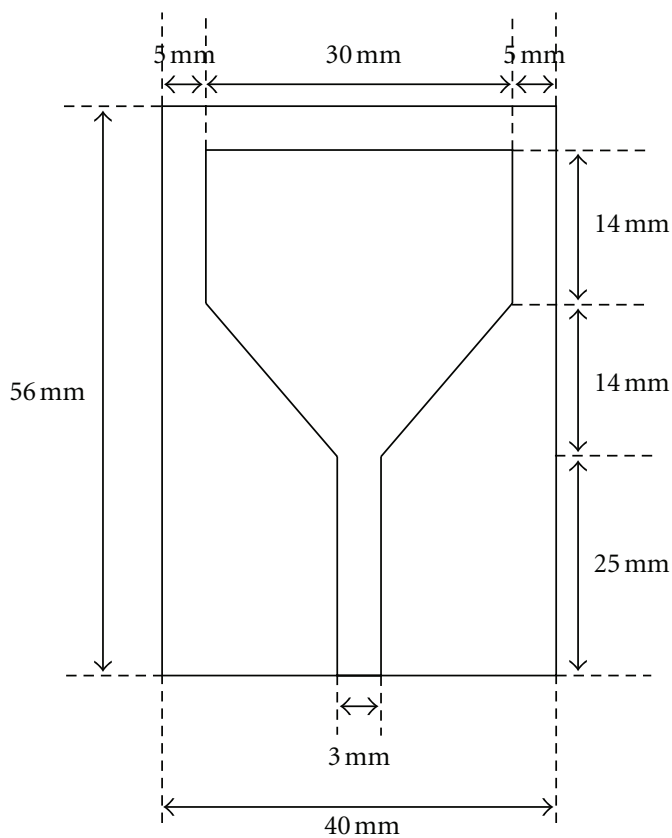

(a)

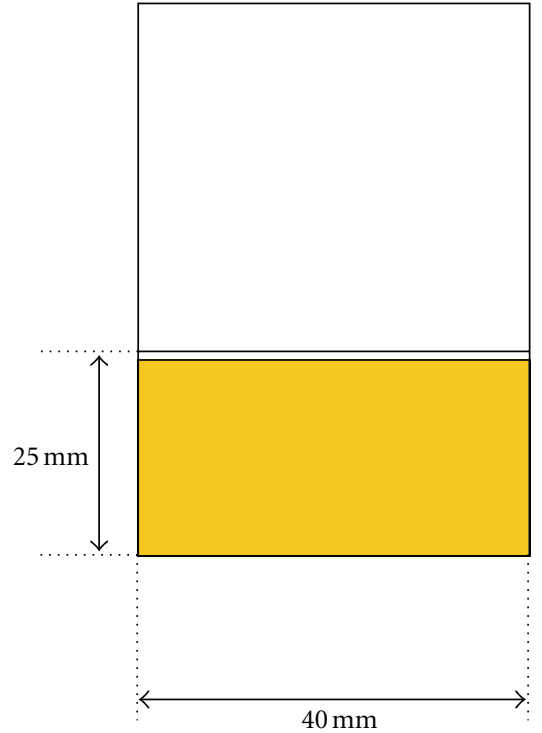

(b)

FIgURE 10: Printed monopole antenna structure at (a) front view and (b) back view.

by control devices. Then, the signals are summed at the combiner device.

\subsection{Antenna}

4.1.1. Single Element. A printed monopole antenna as shown in Figure 10 is chosen to be the antenna element for this paper. The reason is that this type of antenna is compact enough to be arranged to form the array having interelement spacing of half-wavelength. As appeared in Figure 10, the feed line is $25-\mathrm{mm}$ in length, and ground plane is sized by $25 \times 40 \mathrm{~mm}^{2}$. The fabricated antenna is shown in Figure 11 using single-layer FR-4-based board having its thickness of $1.6 \mathrm{~mm}$. This antenna was designed to be able to operate in 1.9-2.4 GHz band. Figure 12 shows the measured return loss of the antenna. As we can see, the antenna provides return loss lower than $-10 \mathrm{~dB}$ within the designated band. Also, its radiation is omnidirectional over the designated band. 


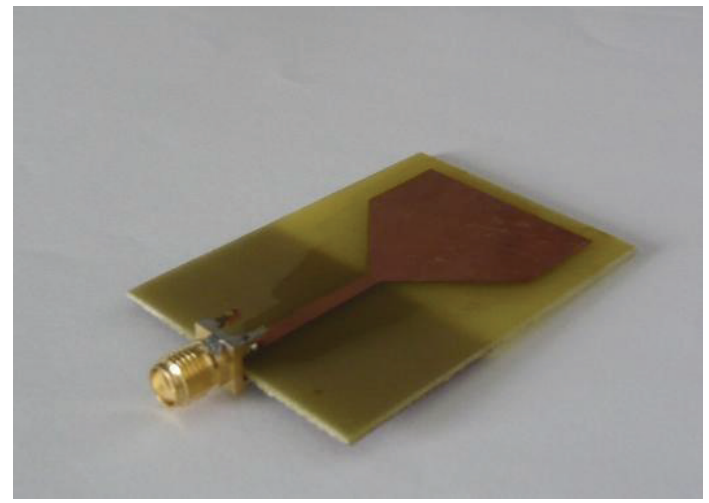

FIGURE 11: Photograph of the printed monopole antenna fabricated on a $40 \times 50 \mathrm{~mm}^{2}$ single-layer FR-4 substrate.

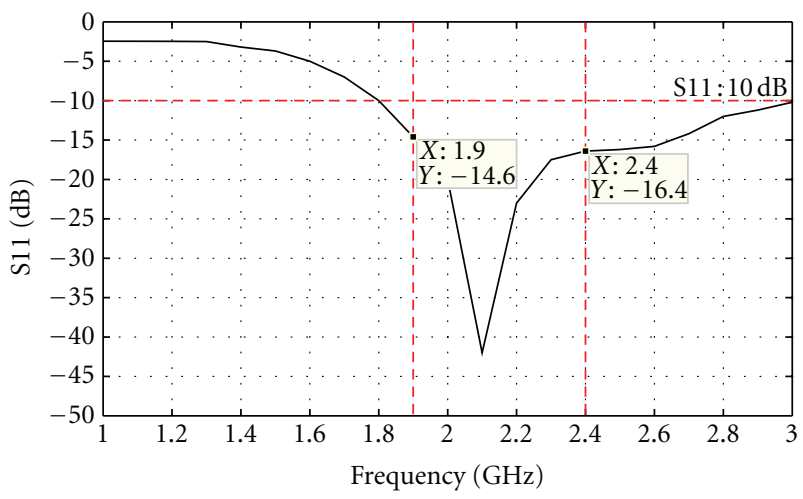

FIGURE 12: Measured return loss of the antenna shown in Figure 11.

4.1.2. Antenna Array. Here we present the design and development of a $4 \times 4$-array antenna which is capable of beam steering in the azimuth direction over an increased operational bandwidth. The design focuses on the frequency band from 1.9 to $2.4 \mathrm{GHz}$. The photograph of constructed antenna array is presented in Figure 13 when the antenna elements are spaced by half-wavelength at $3 \mathrm{GHz}(50 \mathrm{~mm})$, which is higher than the upper frequency of the assumed band $(2.4 \mathrm{GHz})$. The use of this higher frequency $(3 \mathrm{GHz})$ is required in the beamforming algorithm to avoid the edge effect when finding the weighting coefficients.

4.2. Weighting Network. The weighting networks consist of four major components: digital attenuators, digital switching, phase shifters, and power combiners. Each component operates very well over wide range of frequency covering frequencies from 1.9 to $2.4 \mathrm{GHz}$. The detail of each component is shown as follows.

(1) The utilized attenuator is a $50-\Omega$ RF digital step attenuator offering an attenuation range up to $31.5 \mathrm{~dB}$ with $0.5 \mathrm{~dB}$ step controlled by 6 bit serial interface. The operating frequency covers from DC to $2.4 \mathrm{GHz}$.

(2) The digital switching is $50-\Omega$ high isolate SPDT RF switch designed for wireless application, covering a broad

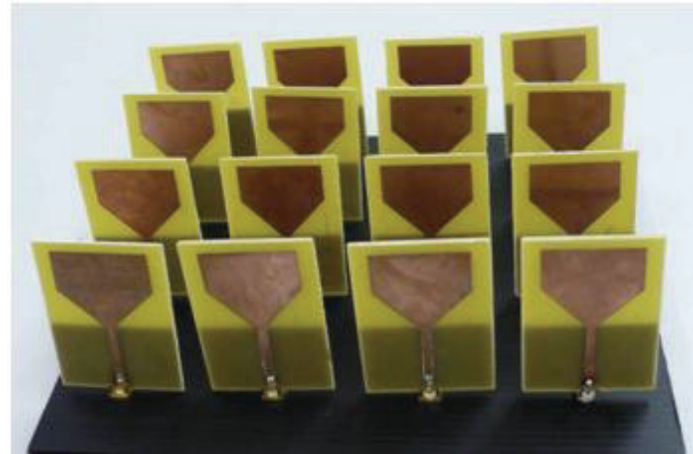

FIGURE 13: Photograph of fabricated $4 \times 4$-array antenna.

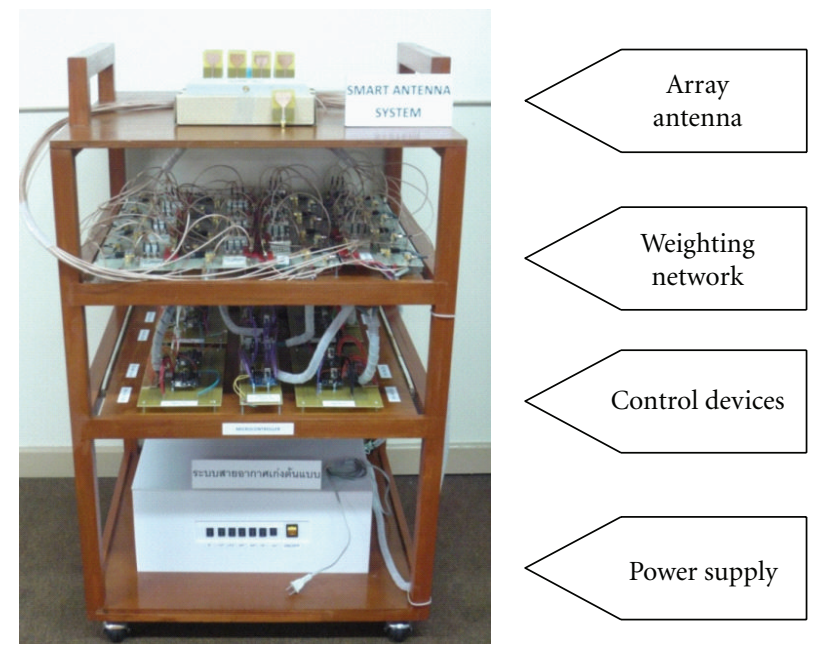

Figure 14: Photograph of full prototype for $4 \times 4$ spatial beamformer consisting of antenna array, weighting network, control devices, and power supply.

frequency range from $\mathrm{DC}$ up to $3 \mathrm{GHz}$ with low insertion loss.

(3) Phase shifters using FR-4 substrate were designed from CST microwave studio. These components are utilized to $180^{\circ}$-shift the phase of signal in order to produce the minus sign for some weighting coefficients.

(4) The power combiners are separated into two different types which are $2: 1$ operating in frequency range from 1.6 to $3.3 \mathrm{GHz}$ and $16: 1$ operating for frequencies from 1.8 to $2.6 \mathrm{GHz}$.

4.3. Control Devices. Twelve ATMEGA328 microprocessors are utilized to digital attenuator and digital switching. The reason to choose this type of control device is that it is low of cost but be able to meet the requirement in this paper.

4.4. Spatial Beamformer. The prototype of $4 \times 4$ spatial beamformer is constructed to test the validation of the proposed method as shown in Figure 14. The power supply is located on the bottom of the shelf. This supports needed power to microcontrollers, digital attenuators, and digital switches. The control devices as mentioned in last section are 


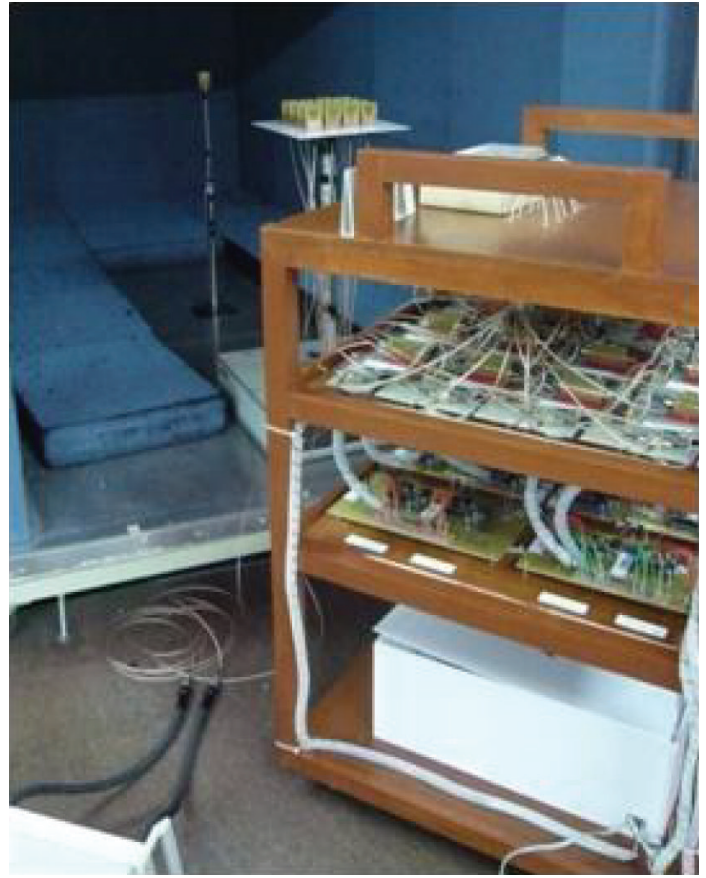

Figure 15: Testing the constructed prototype in an anechoic chamber.

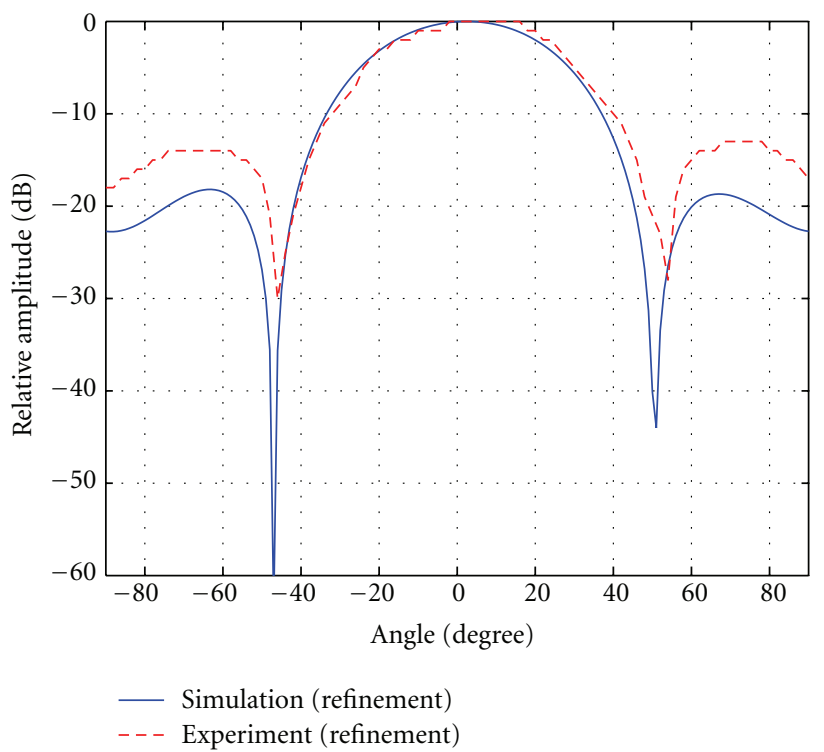

FIGURE 16: Simulated and measured radiation pattern using refinement method for frequency $2.15 \mathrm{GHz}$, when the main beam direction is $7^{\circ}$.

located at the 2 nd shelf followed by the weighting network which is placed on the 3rd shelf as we can see in Figure 14. The top of the shelf is where we put the $4 \times 4$ antenna array as mentioned in last section. Also, the power combiners are placed on the top shelf and pass the output signal to the computer.

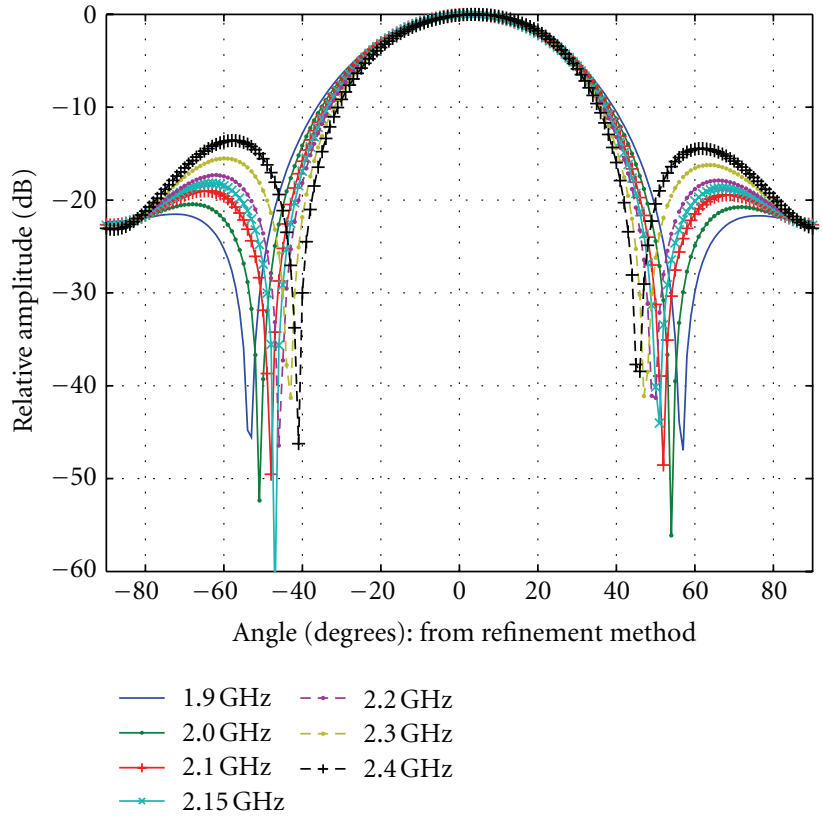

Figure 17: Simulated radiation pattern using refinement method for frequencies from 1.9 to $2.4 \mathrm{GHz}$, when the main beam direction is $7^{\circ}$.

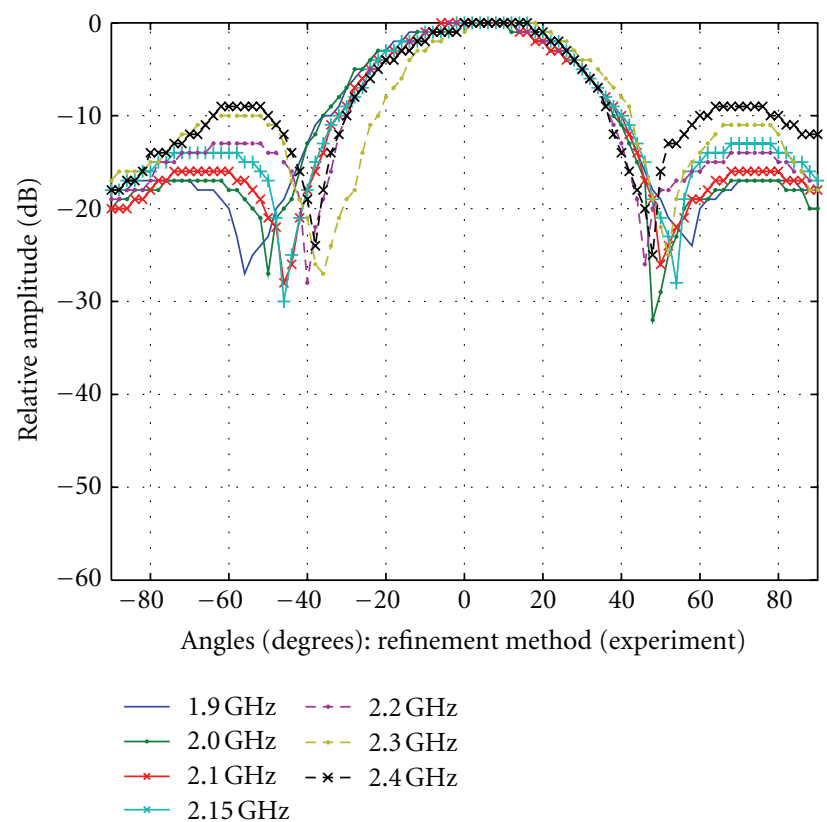

FIGURE 18: Measured radiation pattern using refinement method for frequencies from 1.9 to $2.4 \mathrm{GHz}$, when the main beam direction is $7^{\circ}$.

\section{Experimental Results}

5.1. Experimental Setup. The constructed prototype is tested in anechoic chamber as shown in Figure 15. The obtained results in terms of beamforming performance will be compared to the ones obtained from computer simulation. The measurement is taken place at single frequency from 


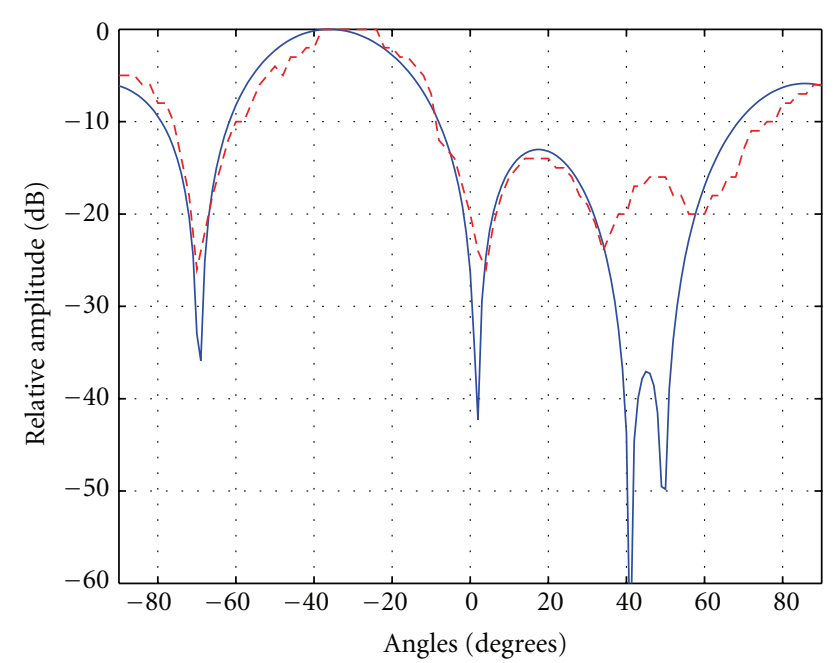

— Simulation (refinement)

- - - Experiment (refinement)

Figure 19: Simulated and measured radiation pattern using refinement method for frequency $2.15 \mathrm{GHz}$, when the main beam direction is $34^{\circ}$.

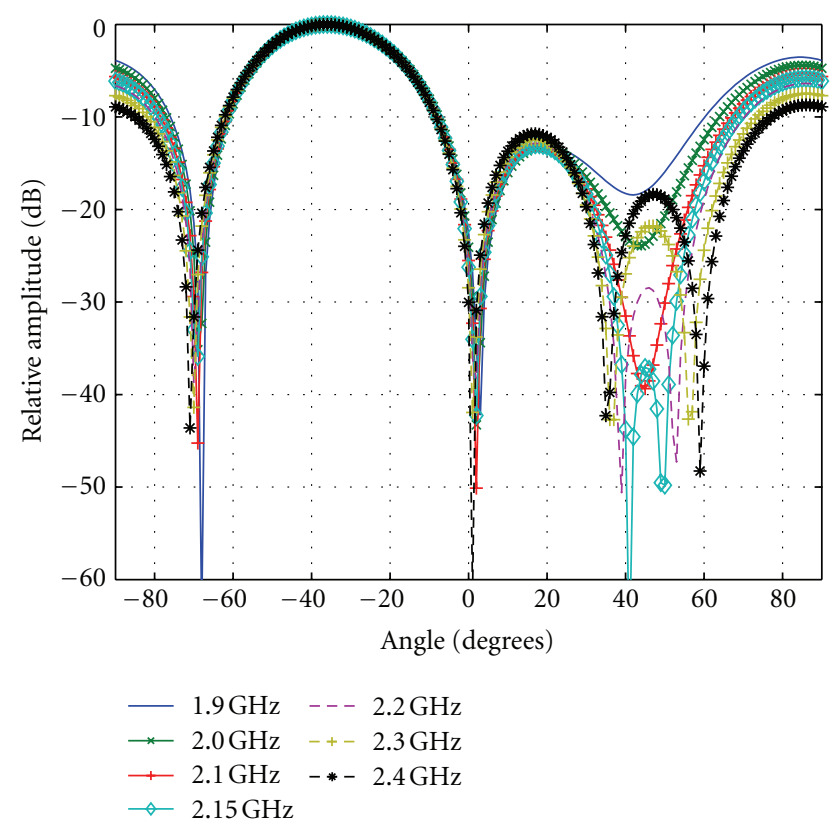

FIGURE 20: Simulated radiation pattern using refinement method for frequencies from 1.9 to $2.4 \mathrm{GHz}$, when the main beam direction is $-34^{\circ}$.

1.9 to $2.4 \mathrm{GHz}$. All the process starting from giving desired mainbeam direction performs automatically. However to show some example, the desired directions to be chosen are 7 directions: $2^{\circ}, 7^{\circ},-17^{\circ}, \pm 34^{\circ}, 57^{\circ}$, and $75^{\circ}$.

5.2. Experimental Results. In this section we validate the beamforming performance of the proposed refinement method through the prototype measurement described in

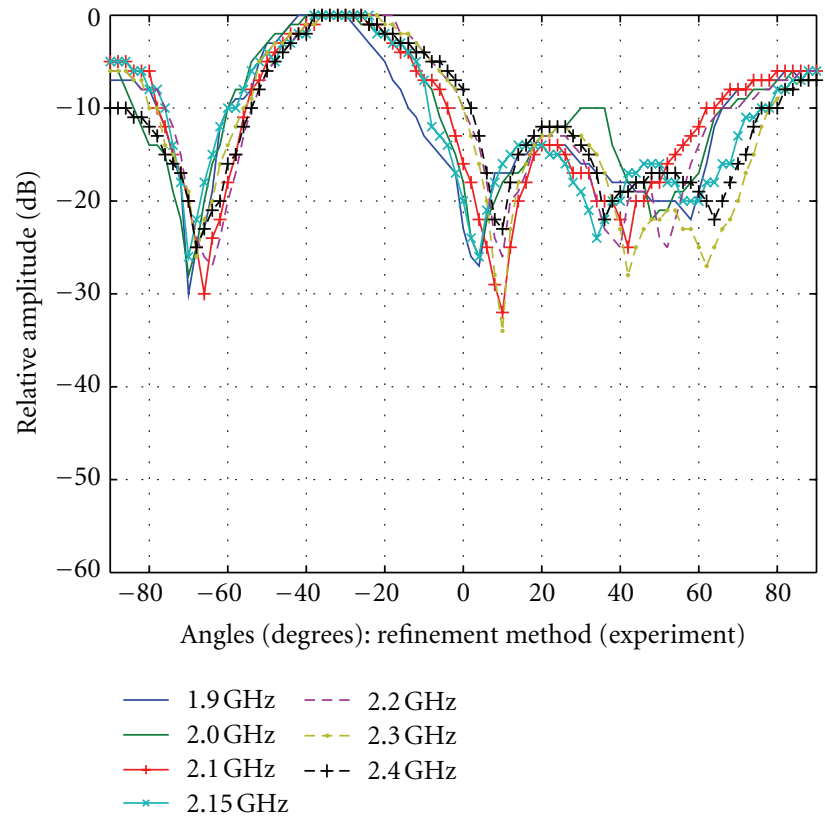

FIGURE 21: Measured radiation pattern using refinement method for frequencies from 1.9 to $2.4 \mathrm{GHz}$, when the main beam direction is $-34^{\circ}$.

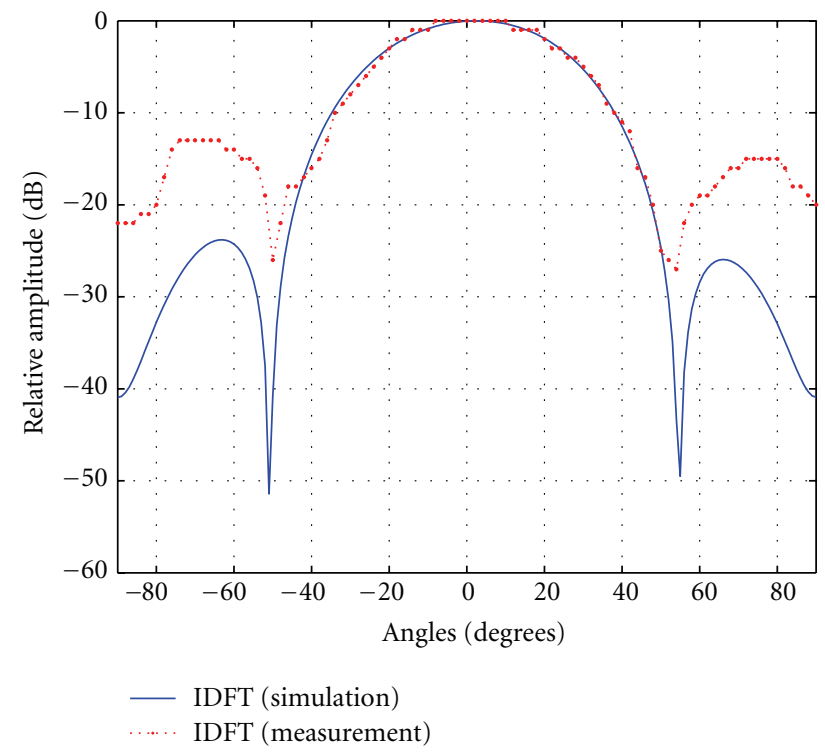

FIGURE 22: Simulated and measured radiation pattern using IDFT method at frequency $2.15 \mathrm{GHz}$, when the main beam direction is $2^{\circ}$.

last section. The performance is compared between employing IDFT method and proposed refinement method. There are 3 cases for this section as follows. Please note that the operating frequencies are assumed from 1.9 to $2.4 \mathrm{GHz}$.

Case A. the beamforming performance using the proposed refinement method is tested. In this case, the mainbeam direction is chosen at $7^{\circ}$. The parameters for this case are as $w_{0}=4 \mathrm{~dB}, \Delta \phi=5^{\circ}, \Delta \mathrm{MLL}=-11 \mathrm{~dB}$, and $\Delta \delta=0^{\circ}$. After 


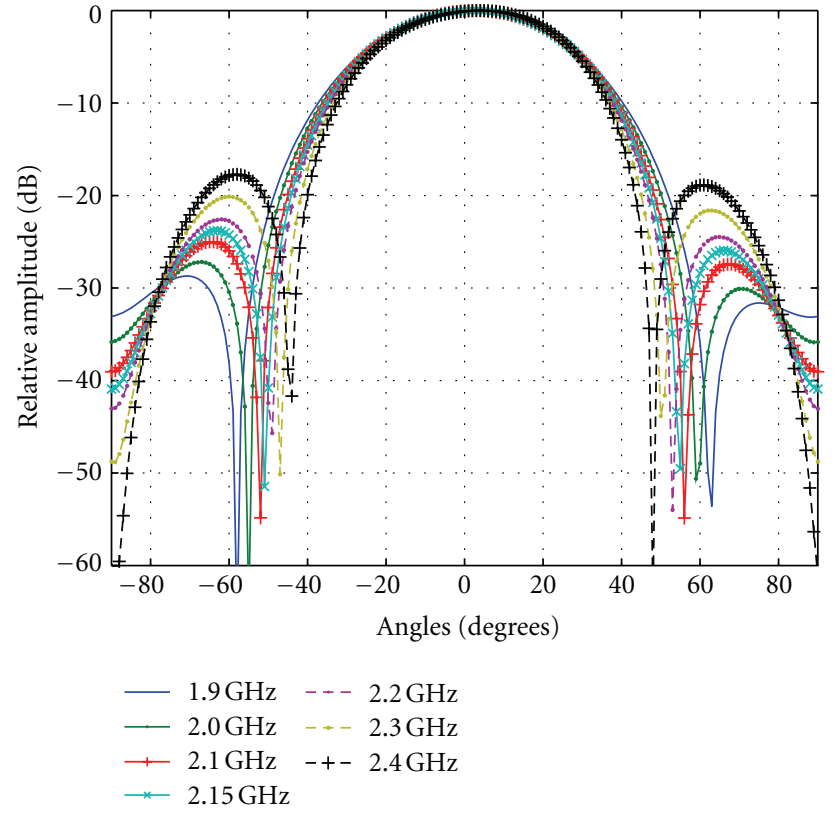

FIGURE 23: Simulated radiation pattern using IDFT method for frequencies from 1.9 to $2.4 \mathrm{GHz}$, when the main beam direction is $2^{\circ}$.

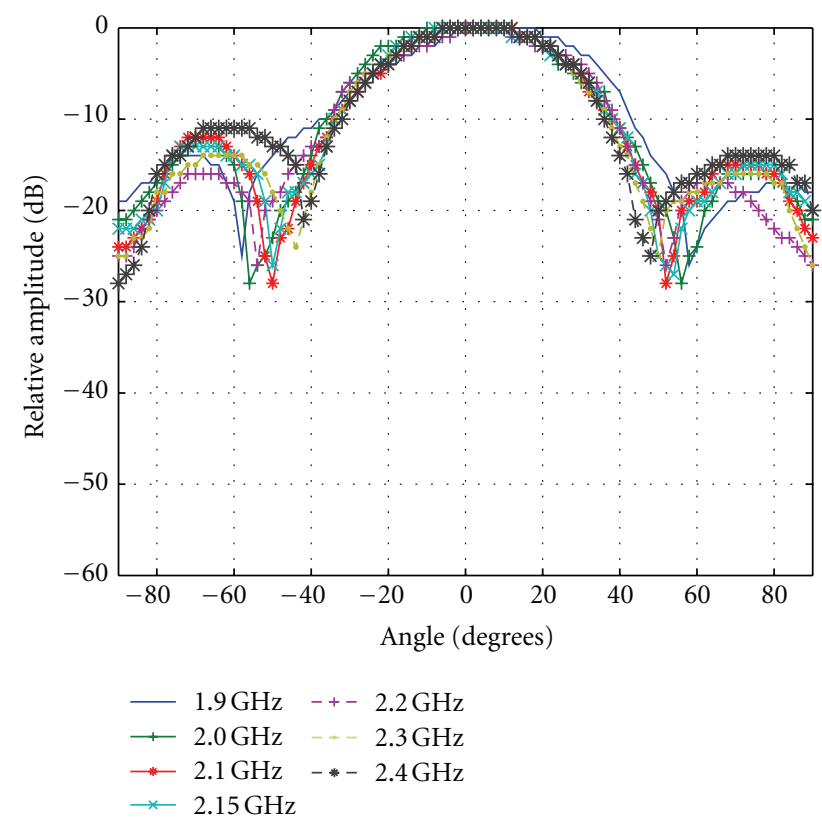

FIGURE 24: Measured radiation pattern using IDFT method for frequencies from 1.9 to $2.4 \mathrm{GHz}$, when the main beam direction is $2^{\circ}$.

the refinement process is done, we have found that the range of attenuation or amplification for weighting coefficients can be reduced from 16 to $4 \mathrm{~dB}$.

The beamforming performance in terms of radiation pattern at frequency $2.15 \mathrm{GHz}$ when using proposed refinement method according to condition for Case $A$ is shown in Figure 16. In the figure, comparison between the result

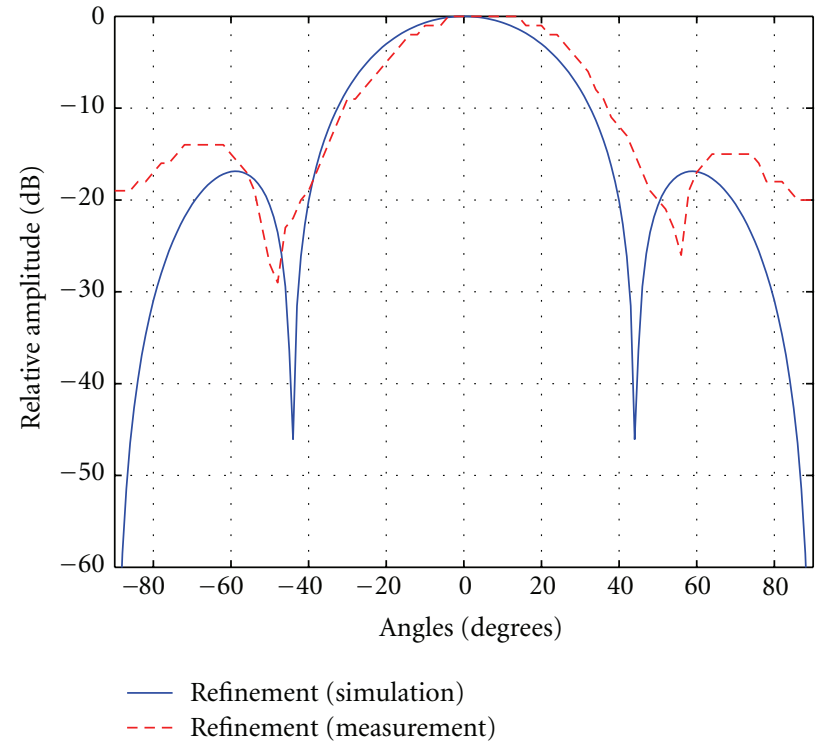

FIGURE 25: Simulated and measured radiation pattern using refinement method at frequency $2.15 \mathrm{GHz}$, when the main beam direction is $2^{\circ}$.

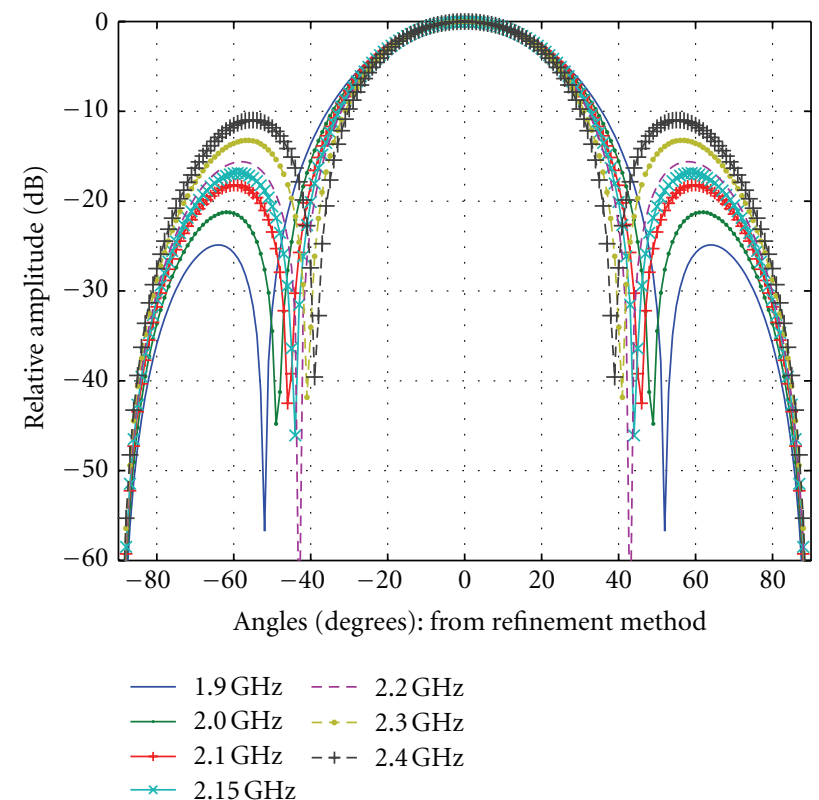

FIGURE 26: Simulated radiation pattern using refinement method for frequencies from 1.9 to $2.4 \mathrm{GHz}$, when the main beam direction is $2^{\circ}$.

obtained from computer simulation and experiment is shown. As we can see, both results have a good agreement to each other. Figure 17 shows the beamforming obtained from simulation for Case A throughout the designated band. We can see that the mainbeam direction does not deviate from the given direction ( $7^{\circ}$ for this case). 


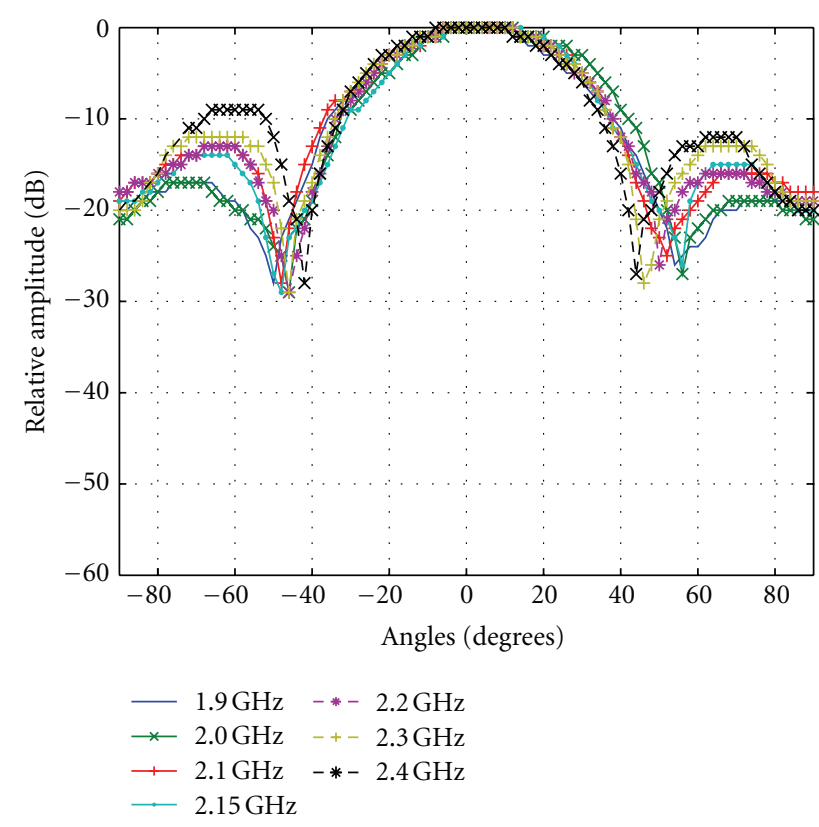

FiguRE 27: Measured radiation pattern using refinement method for frequencies from 1.9 to $2.4 \mathrm{GHz}$, when the main beam direction is $2^{\circ}$.

Case $B$. For this case, the mainbeam is supposed to be pointed to $-34^{\circ}$. Also the parameters for refinement are as follows: $w_{0}=4 \mathrm{~dB}, \Delta \phi=2^{\circ}, \Delta \mathrm{MLL}=-15 \mathrm{~dB}$, and $\Delta \delta=0^{\circ}$. After the refinement process is done, we have found that the range of attenuation or amplification can be reduced from $30 \mathrm{~dB}$ to $4 \mathrm{~dB}$. This is considerable practical.

Figure 19 shows the beamforming performance obtained from simulation and measurement for Case $B$ at frequency $2.15 \mathrm{GHz}$. As we can see, after refinement method is done, the error in mainbeam direction is $2^{\circ}$, and the error in minor lobe level is within $-15 \mathrm{~dB}$. Also, there is no error in beamwidth. This error occurred because we allow some error in the refinement method. Comparing with the results obtained from Case $A$, we can obtain higher range reduction of weighting coefficients while expecting some error in mainbeam direction, minor-lobe level and beamwidth.

Figures 20 and 21 reveal the beamforming performance for Case $B$ throughout the designated band, both from simulation and measurement, respectively. We can observe more error in mainbeam direction, average minor-lobe level and beamwidth compared with the ones presented in Figures 17 and 18. As expected, the ones obtained from measurement are likely to have higher minor-lobe level.

Case C. For this case, the beamforming performance using proposed refinement method comparative with IDFT method is pointed out. The parameters for Case $C$ are as follows: $w_{0}=4 \mathrm{~dB}, \Delta \phi=2^{\circ}, \Delta \mathrm{MLL}=-11 \mathrm{~dB}$, and $\Delta \delta=0^{\circ}$. Please note that the mainbeam direction for this case is changed to $2^{\circ}$. As a result, this case allows us to reduce the range of weighting coefficients from $8 \mathrm{~dB}$ to $4 \mathrm{~dB}$.

Figure 22 shows the beamforming performance at $2.15 \mathrm{GHz}$ using IDFT method for both simulation and measurement. Figures 23 and 24 show radiation pattern throughout the designated band, 1.9 to $2.4 \mathrm{GHz}$, obtained from simulation and measurement, respectively. As we can see, its beamforming behavior looks stable throughout the band. However, the ones obtained from measurement have slightly higher minor lobe level.

Figures 25 to 27 show the performance similar to the ones from Figures 22 to 24 but the proposed refinement method is taken place after we obtain the weighting coefficients from IDFT method. Its beamforming at $2.15 \mathrm{GHz}$ is shown in Figure 25 while Figures 26 and 27 show it performance throughout the designated band via simulation and measurement, respectively. As we can see, the beamforming behavior looks similar to the cases using IDFT method with narrower range of weighting coefficients.

\section{Conclusion}

This paper has presented the method to reduce the range of weighting coefficients for fully spatial beamformer, socalled refinement method. This is considerably useful as the weighting coefficients for this kind of beamformer are real valued; therefore, narrower range of weighting coefficients is advantage. As a result, the choice of attenuators or amplifiers becomes practical. This refinement method is taken place after weighting coefficients are calculated using IDFT method. This proposed method can be succeeded in every mainbeam direction when error in some parameters is allowed. Those parameters are mainbeam direction, average minor lobe level, and beamwidth. The more errors are allowed, the more success cases can be achieved. A full prototype of fully spatial beamformer was constructed to test the validation of the proposed refinement method. The results in terms of radiation pattern obtained from simulation and measurement are compared. The obtained results reveal that we can reduce the range of attenuation or amplification using the proposed refinement method while some significant radiation behaviors are remained.

\section{Acknowledgment}

This work was supported by Suranaree University of Technology (SUT) and by the Office of the Higher Education Commission under NRU project of Thailand.

\section{References}

[1] I. Hamburg, A. Hamburg, M. Gavota, and M. Lazea, "Integrating wireless technology in e-learning for disabled," in Proceedings of the International Conference on Information and Communication Technologies: From Theory to Applications (ICTTA '04), pp. 123-124, April 2004.

[2] M. Ghavami, "An adaptive wideband array using a single real multiplier for each antenna element," in Proceedings of the 13th IEEE International Symposium on Personal, Indoor and Mobile Radio Communications (PIMRC '02), vol. 4, pp. 1805-1809, September 2002.

[3] S. C. Rani, P. V. Subbaiah, and K. C. Reddy, "Smart antenna algorithm for WCDMA mobile communication system," 
International Journal of Computer Science and Network Security, vol. 8, no. 7, 2008.

[4] M. Uthansakul and M. E. Bialkowski, "Impact of wideband signals on smart antenna system," in Proceedings of the 15th International Conference on Microwaves, Radar and Wireless Communications (MIKON '04), vol. 2, pp. 501-504, May 2004.

[5] M. Uthansakul and M. E. Bialkowski, "Frequency-angle dependence compensation of non-uniform components for wideband smart antenna," in Proceedings of the IEEE MITT-S International Microwave Symposium Digest, vol. 2, pp. 12531256, June 2004.

[6] M. Uthansakul and M. E. Bialkowski, "An investigation into smart antenna configurations for wideband communication," in Proceedings of the 15th International Conference on Microwaves, Radar and Wireless Communications (MIKON '04), vol. 2, pp. 505-508, May 2004.

[7] L. Rui, G. Yuchun, Z. Xin, and S. Xiaowei, "An investigation into broadband smart antenna systems for wireless communication," in Proceedings of the International Conference on Microwave and Millimeter Wave Technology (ICMMT'07), pp. 1-4, April 2007.

[8] S. S. Jeon, Y. Wang, Y. Qian, and T. Itoh, "A novel smart antenna system implementation for broad-band wireless communications," IEEE Transactions on Antennas and Propagation, vol. 50, no. 5, pp. 600-606, 2002.

[9] M. Hefnawi and G. Y. Delisle, "Performance analysis of wideband smart antenna systems using different frequency compensation techniques," in Proceedings of the 6th IEEE Symposium on Computers Communications (ISCC '01), pp. 237-243, July 2001.

[10] M. Hefnawi and G. Y. Delisle, "Adaptive array performance with focussing technique," in Proceedings of the IEEE Antennas and Propagation Society International Symposium, vol. 2, pp. 1016-1019, July 1997.

[11] M. Uthansakul and M. E. Bialkowski, "Wideband smart antenna using non-uniform components," in Proceedings of the 15th International Conference on Microwaves, Radar and Wireless Communications (MIKON '04), vol. 3, pp. 1542-1545, May 2004.

[12] M. Ghavami, "Wideband smart antenna theory using rectangular array structures," IEEE Transactions on Signal Processing, vol. 50, no. 9, pp. 2143-2151, 2002.

[13] M. E. Bialkowski and M. Uthansakul, "A wideband array antenna with beam-steering capability using real valued weights," Microwave and Optical Technology Letters, vol. 48, no. 2, pp. 287-291, 2006.

[14] M. Uthansakul and M. E. Bialkowski, "Investigations into a wideband spatial beamformer employing a rectangular array of planar monopoles," IEEE Antennas and Propagation Magazine, vol. 47, no. 5, pp. 91-99, 2005.

[15] M. Uthansakul and M. E. Bialkowski, "Fully spatial wide-band beamforming using rectangular array of planar monopoles," IEEE Transaction on Antenna and Propagation, vol. 54, no. 2, 2006.

[16] M. Uthansakul and M. E. Bialkowski, "A wideband spatial beamformer employing a rectangular array of planar monopoles," in Proceedings of the IEEE Antennas and Propagation Society International Symposium and USNC/URSI Meeting, pp. 303-306, July 2005.

[17] M. Uthansakul and M. E. Bialkowski, "Designing a wideband spatial beamformer for low sidelobe radiation pattern performance," in Proceedings of the Asia-Pacific Microwave Conference (APMC'05), December 2005.
[18] C. Bunsanit, P. Uthansakul, R. Wongsan, and M. Uthansakul, "Low profile multi-beam former operating in wide frequency band," in Proceedings of the International conference on Electrical Engineering/Electrical, Computer, Telecommunication and Information Technology, pp. 774-777, May 2009. 

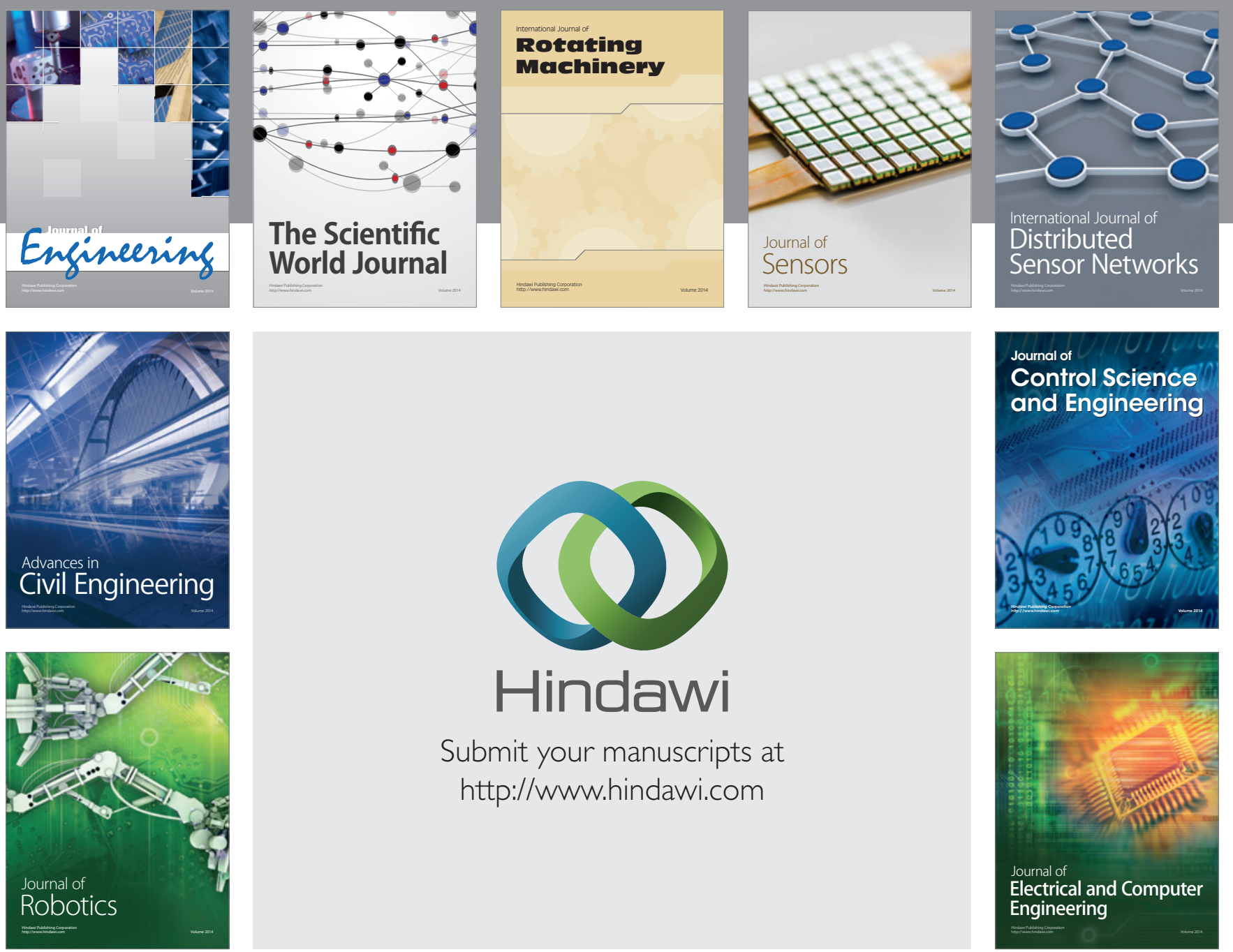

Submit your manuscripts at

http://www.hindawi.com
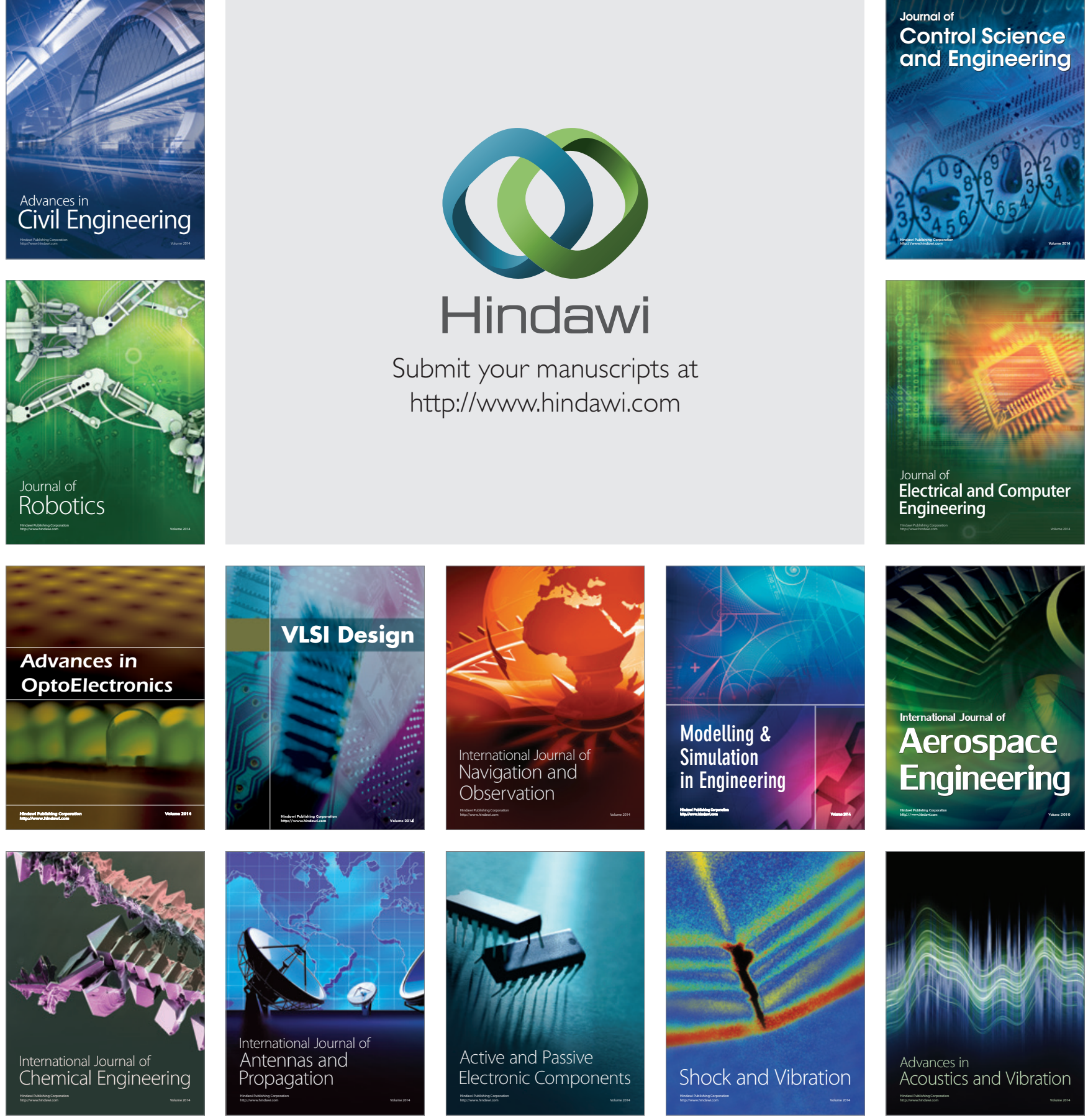